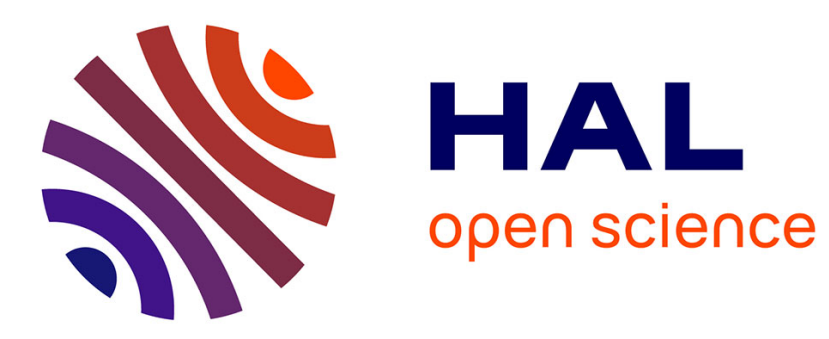

\title{
Model reduction for linear switched systems with autonomous switching
}

Mihaly Petreczky, John Leth, Rasmus Pedersen, Rafael Wisniewski

\section{To cite this version:}

Mihaly Petreczky, John Leth, Rasmus Pedersen, Rafael Wisniewski. Model reduction for linear switched systems with autonomous switching. 2021 American Control Conference (ACC), May 2021, New Orleans (virtual), United States. pp.4465-4471, 10.23919/ACC50511.2021.9483120 . hal03331711

\section{HAL Id: hal-03331711 \\ https://hal.science/hal-03331711}

Submitted on 2 Sep 2021

HAL is a multi-disciplinary open access archive for the deposit and dissemination of scientific research documents, whether they are published or not. The documents may come from teaching and research institutions in France or abroad, or from public or private research centers.
L'archive ouverte pluridisciplinaire HAL, est destinée au dépôt et à la diffusion de documents scientifiques de niveau recherche, publiés ou non, émanant des établissements d'enseignement et de recherche français ou étrangers, des laboratoires publics ou privés. 


\title{
Model reduction for linear switched systems with autonomous switching
}

\author{
Mihály Petreczky, John Leth, Rasmus Pedersen and Rafael Wisniewski
}

\begin{abstract}
This paper proposes a model reduction algorithm for linear switched systems in continuous-time with autonomous switching. An analytic upper bound on the difference between the responses of the original and the reduced order models is derived. The model reduction algorithm is based on the extension of the idea of balanced truncation. The error bound is a direct extension of the one known for linear systems, and it depends on the singular values of the grammians. The class of linear switched systems considered in this paper includes the well-known class of piecewise-linear systems.
\end{abstract}

\section{INTRODUCTION}

Hybrid systems [1]-[3] are a class of nonlinear systems which result from the interaction of continuous-time dynamical subsystems with discrete events. Linear switched systems (LSSs) represent an important class of hybrid systems that consists of a family of linear time-invariant subsystems and a rule that orchestrates the switching between them. More precisely, the state of a linear switched system evolves according to the active linear subsystem. The indices of the linear subsystems are referred to as discrete modes/states, and the signal which determines the index of the active linear system is called the switching signal. Switching signals can be externally generated or functions of the current states of the linear switched system. In the latter case, we speak of linear switched systems with autonomous switching. A particularly popular class of LSSs with autonomous switching are piecewise-linear hybrid systems, where the current discrete mode is a piecewise-linear function of the current continuous state.

There is a rich literature on stability, control and estimation of linear switched systems, see the monographs [1]-[3] and the references therein. However, as a rule, the computational complexity of the proposed methods for analysis, observation and control design grow rapidly with the size of the statespace. In order to address this issue, algorithms for model order reduction (MOR) are required.

Contribution: We propose a model reduction method for linear switched systems with autonomous switching. The class of systems considered in this paper includes the class of piecewise-linear switched systems.

The model reduction method we derive is based on balanced truncation performed simultaneously on all linear subsystems, using a common grammian. The corresponding grammians have to satisfy certain linear matrix inequalities

Mihály Petreczky is with Centre de Recherche en Informatique, Signal et Automatique de Lille (CRIStAL), UMR CNRS 9189, CNRS, Ecole Centrale de Lille, France, mihaly.petreczky@ec-lille.fr

John Leth, Rasmus Pedersen and Rafael Wisneiwski are with the section of Automation and Control, Aalborg University, Fredrik Bajers Vej 7, 9220 Aalborg, Denmark, $\{j j l, r p e, r a f\}$ des.aau.dk
(LMIs). In addition to a novel algorithm, we propose an analytic error bound for the difference between the input-output behaviors of the original and the reduced-order models. This error bound is a direct counterpart of the well-known error bound for balanced truncation of linear systems [4], and it involves the singular values of the grammians.

Related work: To the best of our knowledge, the contribution of the paper is new. Indeed, most of the existing methods for model reduction of hybrid system deal with switched systems for which the switching was an external input or stochastic, these methods are either based on LMIs [5]-[8], balanced truncation [9]-[20], or moment matching [21]-[24]. To the best of our knowledge, the only results on model reduction of linear switched system with autonomous switching are [25]-[29]. In [29] an observability reduction algorithm was proposed, such that the reduced model has exactly the same input-output behavior as the original one. In [25], a model reduction algorithm based on balanced truncation of each subsystem using a different grammian was proposed, but no error bound was formulated. In contrast to [25], the current paper provides an error bound, and the proposed model reduction procedure is based on balanced truncation using the common grammians. In [26], [27], a model reduction procedure which guarantees that the reduced model has the same steady-state output response to certain inputs as the original model. In contrast, the current paper proposes error bounds for all finite energy inputs. The results of [28] are the closest to those of the current paper. Indeed, the approach taken in this paper is inspired by that of [28]. However, in contrast to [28], we do not require the piecewise-linear map describing the dynamics of the LSS to be continuous. This latter compels us to use Fillipov solutions. In a way, the current paper can be viewed as an extension of the results of [28] to a more general class of linear switched systems. It is remarked that the obtained error bounds are identical to the error bounds obtained for linear systems [30] and for LSSs with external switching [13], [16].

Outline: Section II introduces the notation used in this paper. In Section III we formulate the notion of a LSS, discuss the notion of a solution to an LSS and explain what it means for a LSS to be balanced. Section IV contains the main contribution in the form of an algorithm for balanced truncation together with an analytic error bound. Section V comprises an example illustrating the findings of this paper.

\section{NOTATION}

For a set $S$, we let $2^{S},|S|$ and $\bar{S}$ denote the power set of $S$, the cardinality of $S$, and the closure of $S$. respectively. The set of natural numbers including 0 is denoted $\mathbb{N}$, and $T=\mathbb{R}_{+}$ 
indicates the set of non-negative reals. The Euclidean 2norm is written as $\|\cdot\|$ and $\mathbb{R}^{k \times l}$ denotes the set of all $k \times l$ matrices with real entries. For a symmetric matrix definiteness is indicated by $>, \geq,<$ and $\leq$.

A map $f: T \rightarrow \mathbb{R}^{n}$ is piecewise-continuous, if $f$ has finitely many points of discontinuity on any compact subinterval of $T$, and at any point of discontinuity the lefthand and right-hand side limits of $f$ exist, i.e., are finite. Let $C\left(T, \mathbb{R}^{n}\right)$ denote the set of all continuous maps, let $P C\left(T, \mathbb{R}^{n}\right)$ denote the set of all piecewise-continuous maps and $A C\left(T, \mathbb{R}^{n}\right)$ denote the set of all absolutely continuous maps $f: T \rightarrow \mathbb{R}^{n}$. Let $L_{2}\left(T, \mathbb{R}^{n}\right)$ denote the Hilbert space of all Lebesgue measurable maps $f: T \rightarrow \mathbb{R}^{n}$ with finite $L_{2}$-norm $\|f\|_{2}=\sqrt{\int_{0}^{\infty}\|f(s)\|^{2} d s}<\infty$, and let $L_{\infty}\left(T, \mathbb{R}^{n}\right)$ denote the set of all Lebesque measurable essentially bounded maps $f: T \rightarrow \mathbb{R}^{n}$.

In the sequel, the following shorthand notation and terminology will be used: $X=\mathbb{R}^{n}$ will be referred to as the state space, $Y=\mathbb{R}^{p}$ and $Z=\mathbb{R}^{p^{\prime}}$ will be referred to as output spaces, and $U=\mathbb{R}^{m}$ will be referred to as the input space. Moreover, $\mathcal{U}, \mathcal{Q}, \mathcal{X}, \mathcal{Y}$ and $\mathcal{Z}$ will denote $L_{2}(T, U) \cap C(T, U) \cap L_{\infty}(T, U), P C(T, Q), A C(T, X)$, $P C(T, Y)$ and $P C(T, Z)$, respectively.

\section{LINEAR SWITCHED SYSTEMS}

Below, we formulate the definition of linear switched systems and their system theoretic properties. The presentation is partially based on [31]-[33].

Definition 1 (Linear switched system): A linear switched system with internal switching (abbreviated as LSS) is a tuple

$$
\Sigma(C)=\left(n, Q,\left\{\left(A_{q}, B, C\right) \mid q \in Q\right\}, \phi\right)
$$

where $Q=\mathbb{N} \backslash\{0\}$ or $Q=\{1, \ldots, D\}$ for some fixed $D \in$ $\mathbb{N} \backslash\{0\}, A_{q} \in \mathbb{R}^{n \times n}$ for each $q \in Q, B \in \mathbb{R}^{n \times m}, C \in \mathbb{R}^{p \times n}$, and $\phi: X \rightarrow 2^{Q}$. The number $n \in \mathbb{N}$, sometimes denoted $\operatorname{dim} \Sigma$, is called the dimension of the LSS $\Sigma$. Elements of $Q$ will be called discrete modes and $\phi$ will be referred to as the partition map.

For each $q \in Q$, the triple $\left(A_{q}, B, C\right)$ represent an inputoutput system on the state space form:

$$
\dot{x}=A_{q} x+B u, \quad y=C x .
$$

Moreover, the phrases "internal switching" and "partition map" refer to the fact that the discrete modes $q \in Q$ are assigned internally through a ( $\phi$ induced) partitioning of the state space $X$ given by the family $\mathcal{P}=\left\{\phi^{-1}(S)\right\}_{S \in 2^{Q}}$ of subsets of $X$. Note that for given $\phi$, we may obtain a partitioning of the output space $Y$ from the partitioning of $X$ if there exists $\psi: Y \rightarrow 2^{Q}$ such that $\phi(x)=\psi(C x)$.

Remark 1: A linear switched system $\Sigma(C)$ with external switching, as defined in [1], can be obtained from Definition 1 (with $\Sigma=\Sigma(C)$ ) by leaving out the partition map $\phi$ thus obtaining a situation where the discrete modes $q$ could be chosen externally contrary to the case here where the discrete modes $q$ are given in terms of the state feedback strategy $q=\phi(x)$.
In this paper, $\phi$ will not be required to have any specific properties (see however Theorem 1 and the related comments, and Remark 3. This allows very general partitions. When used in a particular application, one typically needs to enforce various regularity conditions, e.g., the family of subsets $\mathcal{P}$ has non-empty and non-overlapping interior, or even more specific that $\mathcal{P}$ is a piecewise linear partitioning as defined in [34]. To exemplify the map $\phi$, we define the following "polynomial" partition. Let $p_{1}=2 x_{1}^{2}+x_{2}^{2}$ and $p_{2}=x_{1}^{2}+2 x_{2}^{2}$, and define

$$
\phi(x)=\left\{\begin{array}{l}
1 \quad \text { if } p_{1} \leq 1 \text { and } p_{2} \leq 1 \\
2 \quad \text { if } p_{1} \leq 1 \text { and } p_{2} \geq 1 \\
3 \quad \text { if } p_{1} \geq 2 \text { and } p_{2} \leq 1 \\
4 \text { if } p_{1} \geq 2 \text { and } p_{2} \geq 1
\end{array} .\right.
$$

The partition induced by $\phi$ is depicted in Fig. 1. In this case, $Q=\{1,2,3,4\},\left\{\phi^{-1}(\{q\})\right\}_{q \in Q}$ covers $X$. The sets $\phi^{-1}(\{i\}), i=1,4$ consist of one path connected subsets of $X$ and $\phi^{-1}(\{i\}), i=2,3$ consist of two path connected subsets of $Y$. Moreover, $\phi$ is truly set-valued, e.g., $\phi(\sqrt{ }(1 / 3), \sqrt{(1 / 3)})=\{1,2,3,4\}$. Note that to avoid $\phi^{-1}(\{q\})$ being disconnected one can include the sets $\left\{x_{1}=\right.$ $0\}$ and $\left\{x_{2}=0\right\}$ into the partitioning (inducing $D=16$ path connected partitions) as depicted on the right in Fig. 1 .
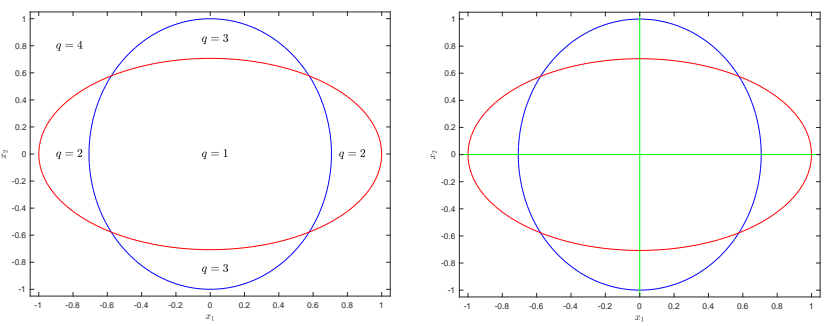

Fig. 1. Partitioning: $p_{1}=1$ (blue ellipse), $p_{2}=1$ (red ellipse).

Definition 2 (Solution): A solution of the switched system (with internal switching) $\Sigma$ is a tuple $(x, y, u)$ of signals $u \in \mathcal{U} 1, x \in \mathcal{X}, y \in \mathcal{Y}$ that satisfy

$$
\dot{x}(t) \in f(x(t), u(t)), \quad y(t)=C x(t)
$$

with $f: X \times U \rightarrow 2^{X}$ given by

$$
f(x, u)=\operatorname{co}\left\{v \in X \mid v=A_{q} x+B u, q \in \phi(x)\right\} .
$$

and $\operatorname{co}(S)$ denoting the convex hull of the subset $S \subset X$. We shall call $u=u(\cdot) \in \mathcal{U}$ the control input, $x=x(\cdot) \in \mathcal{X}$ the state trajectory, and $y=y(\cdot) \in \mathcal{Y}$ the output trajectory.

As mentioned in Remark 1, we may consider $\phi$ as a setvalued state feedback. That is, for each state $x$, the partition map $\phi$ specifies the discrete states $q \in \phi(x)$ and therefore dynamical systems which contribute to the evolution of the LSS given by (2).

\footnotetext{
${ }^{1}$ Note that the elements of $\mathcal{U}$ are continuous in $t$, are bounded and have finite energy; we conjecture that the results of the paper can be extended to the case of general measurable inputs from $L_{2}(T, U)$, but in order to avoid technical complications, we prefer to stick to bounded and continuous control inputs
} 
We will need the following result.

Theorem 1: Let $\Sigma$ be a LSS and assume that the partition map $\phi$ is such that the set-valued map

$$
G(x, t)=\left\{v \in X \mid v=A_{q} x+B u(t), q \in \phi(x)\right\}
$$

is upper semi-continuous and bounded ${ }^{2}$ for every $u \in \mathcal{U}$. Then for every $x_{0} \in X$ and $u \in \mathcal{U}$, there exists a solution $(x, y, u)$ of $\Sigma$ such that $x(0)=x_{0}$.

Specifically, $G$ in (4) is upper semi-continuous if $\phi$ generates a partitioning with subsets having non-empty and non-overlapping interior, and such that whenever $R=$ $\overline{\phi^{-1}(\{q\})} \cap \overline{\phi^{-1}\left(\left\{q^{\prime}\right\}\right)} \neq \emptyset$ then $\left\{q, q^{\prime}\right\} \subset \phi(x)$ for all $x \in R$. This condition is similar to the construction of a piecewise linear partitioning defined in [34]. In particular, the partition map $\phi$ from (1) generates a upper semi-continuous map $G$. Note however, that if any of the non-strict polynomial inequalities (for example, $p_{1} \leq 1$ in the first line of (1)) is substituted by a strict inequality, $G$ would not be upper semi-continuous. The requirement that $G$ is bounded is somewhat more problematic. Intuitively, an example when $G$ is bounded if for large enough $x$, for all $q \in \phi(x), A_{q}=0$, i.e., the vector fields outside a bounded set do not depend on $x$. This is a restrictive condition, but it is often true in practice. If $G$ is not bounded, then solutions of the system will still exist, but not necessarily on the whole time axis $T$.

Proof: Let $u=u(t) \in \mathcal{U}$ be given, and note that $F(x, t)=f(x, u(t))$ is non-empty, convex and compact for each $(x, t) \in X \times T$. Moreover, since $G$ from (4) is upper semi-continuous it follows that $F(x, t)=\operatorname{co}\{G(x, t)\}$ is upper semi-continuous by Lemma 16 in [35, p. 66]. Since $G(x, t)$ is bounded, $F(x, t)$ is bounded too, hence there exists a compact set such that $F(x, t)$ is a subset of that compact set for all $(x, t)$. Hence by Theorem 4 in [36, p. 101, Chapter 2] the proof is complete.

In the sequel, in order to avoid technical problems with existence of a solution defined on the whole time axis $T$, we will consider well-posed systems, which are defined as follows:

Definition 3 (Well-posed LSS): A LSS $\Sigma$ is said to be well-posed, if the set-valued map $G$ from (4) is upper semicontinuous and bounded for all $u \in \mathcal{U}$.

Remark 2 (Boundedness of $G$ ): We conjecture that the results of the paper can be extended to systems which are not well-posed. In particular, if we drop the condition that $G$ is bounded, then we can still guarantee existence of a solution, but not necessarily on the whole time axis $T$. We believe that the main results of the paper can still be stated, but their formulation would become more cumbersome, due to the necessity to deal with the domain of definition of various solutions.

Definition 4 (Observability grammian): An observability grammian of a LSS $\Sigma$ is a matrix $\mathcal{Q}>0$ such that for all $\left(\zeta_{1}, \zeta_{2}\right) \in X \times X$, and $q_{i} \in \phi\left(\zeta_{i}\right), i=1,2$

$$
\begin{array}{rl}
\left(A_{q_{1}} \zeta_{1}-A_{q_{2}} \zeta_{2}\right)^{T} & \mathcal{Q}\left(\zeta_{1}-\zeta_{2}\right) \\
+ & 0.5\left(\zeta_{1}-\zeta_{2}\right)^{T} C^{T} C\left(\zeta_{1}-\zeta_{2}\right)<0 .
\end{array}
$$

${ }^{2}$ Recall that $G$ is bounded if there exists $M$ such that for all $x, t$ and $v \in G(x, t),\|v\|_{2} \leq M$.
Definition 5 (Controllability grammian): An controllability grammian of a LSS $\Sigma$ is a matrix $\mathcal{P}>0$ such that for all $\left(\zeta_{1}, \zeta_{2}, u\right) \in X \times X \times U \backslash\{0\}$, and $q_{i} \in \phi\left(\zeta_{i}\right), i=1,2$

$$
\left(A_{q_{1}} \zeta_{1}+A_{q_{2}} \zeta_{2}+2 B u\right)^{T} \mathcal{P}^{-1}\left(\zeta_{1}+\zeta_{2}\right)-\|2 u\|^{2}<0 .
$$

A LSS $\Sigma$ is said to be balanced, if it has observability and controllability grammians which are diagonal and equal, i.e., there exists a diagonal matrix $\Lambda>0$ such that $\Lambda$ is an observability and a controllability grammian of $\Sigma$.

Remark 3: Computing an observability or a controllability grammian can be formulated as an LMI. At the outset, we notice that (5) can be formulated as $y^{T} R_{O} y^{\prime}-r_{0} \geq 0$ for non-zero $y^{\prime}=\left(\zeta_{1}, \zeta_{2}\right)$, and for some $r_{0}>0\left(r_{0}\right.$ is used here to change the strict to non-strict inequality), and for the symmetric matrix $R_{O}=R_{O}(\mathcal{Q})$ given by

$$
-\left[\begin{array}{cc}
A_{q_{1}}^{T} \mathcal{Q}+\mathcal{Q} A_{q_{1}}+C^{T} C & -A_{q_{1}}^{T} \mathcal{Q}-\mathcal{Q} A_{q_{2}}-C^{T} C \\
\star & A_{q_{2}}^{T} \mathcal{Q}+\mathcal{Q} A_{q_{2}}+C^{T} C
\end{array}\right] .
$$

Similarly, 6) can be formulated as $y^{T} R_{C} y-r_{0} \geq 0$ for non-zero $y=\left(\zeta_{1}, \zeta_{2}, u\right)$ and $R_{C}=R_{C}\left(\mathcal{P}^{-1}\right)=R_{C}(\mathcal{R})$ given by

$$
-\left[\begin{array}{ccc}
A_{q_{1}}^{T} \mathcal{R}+\mathcal{R} A_{q_{1}} & A_{q_{1}}^{T} \mathcal{R}+\mathcal{R} A_{q_{2}} & 2 \mathcal{R} B \\
\star & A_{q_{2}}^{T} \mathcal{R}+\mathcal{R} A_{q_{2}} & 2 \mathcal{R} B \\
\star & \star & -8
\end{array}\right] .
$$

If the partition map $\phi$ is of a particular form defined below then the S-procedure applies. Specifically, for each $q \in Q$, let $\phi$ be such that $q \in \phi(\zeta)$ if and only if there exist $H_{k}(q) \in$ $\mathbb{R}^{n \times n}, h_{k}(q) \in \mathbb{R}^{n}$ and $a_{k}(q) \in \mathbb{R}(k=1, \ldots, l, l=l(q))$ such that

$$
\begin{aligned}
& \{\zeta \in X \mid q \in \phi(\zeta)\} \\
= & \left\{\zeta \in X \mid \zeta^{T} H_{k}(q) \zeta+h_{k}^{T}(q) \zeta+a_{k}(q) \geq 0, k=1, \ldots l\right\} .
\end{aligned}
$$

Subsequently, checking the controllability and observability grammian conditions in (5) and (6) reduces to computing $\mathcal{Q}$ and $\mathcal{R}=\mathcal{P}^{-1}$ such that

$$
\left[\begin{array}{cc}
R_{a} & 0 \\
0 & r_{0}
\end{array}\right]+\gamma_{1}\left[\begin{array}{cc}
R_{1 k} & s_{1 k} \\
s_{1 k}^{T} & r_{1 k}
\end{array}\right]+\gamma_{2 k}\left[\begin{array}{cc}
R_{2 k} & s_{2 k} \\
s_{2 k}^{T} & r_{2 k}
\end{array}\right] \geq 0
$$

with $a \in\{O, C\}$ for some $\gamma_{1} \geq 0$ and $\gamma_{2} \geq 0$, where for $a=C$

$$
\begin{aligned}
R_{1 k} & =\left[\begin{array}{ccc}
H_{k}\left(q_{1}\right) & 0 & 0 \\
0 & 0 & 0 \\
0 & 0 & 0
\end{array}\right], R_{2 k}=\left[\begin{array}{ccc}
0 & 0 & 0 \\
0 & H_{k}\left(q_{2}\right) & 0 \\
0 & 0 & 0
\end{array}\right], \\
s_{1 k} & =\left[\begin{array}{lll}
h_{k}\left(q_{1}\right)^{T} & 0 & 0
\end{array}\right]^{T}, s_{2 k}=\left[\begin{array}{lll}
0 & h_{k}\left(q_{2}\right)^{T} & 0
\end{array}\right]^{T}, \\
r_{i k} & =a_{k}\left(q_{i}\right)
\end{aligned}
$$

for all $\left(q_{1}, q_{2}\right) \in Q \times Q$. In the case of observability $(a=O)$, one should remove the last row and column of $R_{1}$ and $R_{2}$, and the last element of $s_{1}$ and $s_{2}$.

For the example described by (1), let $P_{i}$ be the matrix corresponding to $p_{i}$. We have $l(q)=2$,

$$
\begin{aligned}
& H_{1}(q=1)=-P_{1}, H_{2}(q=1)=-P_{2}, \\
& h_{1}(q=1)=h_{2}(q=1)=0, a_{1}(q=1)=a_{2}(q=1)=1
\end{aligned}
$$

describing $\{\zeta \in X \mid 1 \in \phi(\zeta)\}$, similarly for the remaining 6 sets. 


\section{BALANCED TRUNCATION AND ERROR BOUND}

In the sequel, we present the model reduction algorithm (Procedure 1) followed by the main result (Theorem 2) giving an upper bound on the difference between the output of the original and the reduced order system.

Procedure 1: Balanced truncation. Consider a well posed LSS $\Sigma=\left(n, Q,\left\{\left(A_{q}, B, C\right) \mid q \in Q\right\}, \phi\right)$.

1) Find a positive definite solution $\mathcal{Q}>0$ to $(5)$.

2) Find a positive definite solution $\mathcal{P}>0$ to 6 .

3) Find $U$ such that $\mathcal{P}=U U^{T}$ and find an orthogonal $K$ such that $U^{T} \mathcal{Q} U=K \Lambda^{2} K^{T}$, where $\Lambda$ is diagonal with the diagonal elements taken in decreasing order. Define the transformation $\mathcal{S}=\Lambda^{1 / 2} K^{T} U^{-1}$.

4) Replace $\Sigma$ with

$$
\begin{aligned}
& \Sigma_{\text {bal }}= \\
& \left(n, Q,\left\{\bar{A}_{q}=\mathcal{S} A_{q} \mathcal{S}^{-1}, \bar{B}=\mathcal{S} B, \bar{C}=C \mathcal{S}^{-1}\right\}_{q \in Q}, \phi_{b a l}\right)
\end{aligned}
$$

where $\phi_{\text {bal }}(\zeta)=\phi(\mathcal{S} \zeta)$.

5) The transformed system $\Sigma_{\text {bal }}$ is balanced. i.e., $\Lambda=$ $\left(\mathcal{S}^{-1}\right)^{T} \mathcal{Q S}^{-1}=\mathcal{S} \mathcal{P S}^{T}$ is both and observability and controllability grammian.

6) Assume that $\Lambda=\operatorname{diag}\left(\sigma_{1}, \ldots, \sigma_{n}\right), \sigma_{1} \geq \sigma_{2} \geq \cdots \geq$ $\sigma_{n}$. Choose $r<n$ and let $\Lambda_{1}=\operatorname{diag}\left(\sigma_{1}, \ldots, \sigma_{r}\right)$. Choose $\hat{A}_{q} \in \mathbb{R}^{r \times r}, \hat{B} \in \mathbb{R}^{r \times m}$ and $\hat{C} \in \mathbb{R}^{p \times r}$ so that

$$
\bar{A}_{q}=\left[\begin{array}{cc}
\hat{A}_{q} & A_{q, 12} \\
A_{q, 21} & A_{q, 22}
\end{array}\right], \quad \bar{B}=\left[\begin{array}{c}
\hat{B} \\
B_{2}
\end{array}\right], \bar{C}^{T}=\left[\begin{array}{l}
\hat{C}^{T} \\
C_{2}^{T}
\end{array}\right] .
$$

Return as a reduced order model $\hat{\Sigma}=$ $\left(r, Q,\left\{\left(\hat{A}_{q}, \hat{B}, \hat{C}\right) \mid q \in Q\right\}, \hat{\phi}\right)$, where $\hat{\phi}(\zeta)=\phi(\zeta, 0)$.

Theorem 2 (Error bound): Let $\hat{\Sigma}$ be the LSS returned by Procedure 11 Then $\hat{\Sigma}$ is well posed, balanced, and for any solution $(x, y, u)$ of $\Sigma$ and for any solution $(\hat{x}, \hat{y}, u)$ of $\hat{\Sigma}$, such that $x(0)=0, \hat{x}(0)=0$,

$$
\|y-\hat{y}\|_{L_{2}} \leq\left(2 \sum_{i=r+1}^{n} \sigma_{i}\right)\|u\|_{L_{2}} .
$$

The proof of Theorem 2 relies on proving its statement for the case of balanced $\Sigma$ and for $r=n-1$, i.e. when only one state is discarded (Theorem 3 below).

More precisely, assume that $\Sigma$ is a well posed and balanced LSS with a (common) grammian $\Lambda=$ $\operatorname{diag}\left(\sigma_{1}, \ldots, \sigma_{n}\right)$ and set $r=n-1$. Then $\mathcal{S}$ in Procedure 1 can be chosen to be identity and the parameters of $\hat{\Sigma}$ will satisfy

$$
\begin{aligned}
& A_{q}=\left[\begin{array}{cc}
\hat{A}_{q} & A_{q, 12} \\
A_{q, 21} & A_{q, 22}
\end{array}\right], \quad B=\left[\begin{array}{c}
\hat{B} \\
B_{2}
\end{array}\right], \\
& C=\left[\begin{array}{ll}
\hat{C} & C_{2}
\end{array}\right], \quad \Lambda=\left[\begin{array}{cc}
\Lambda_{1} & 0 \\
0 & \sigma_{n}
\end{array}\right], \\
& \hat{\phi}(\zeta)=\phi(\zeta, 0) .
\end{aligned}
$$

Theorem 3: The LSS $\hat{\Sigma}$ is balanced and well-posed, and for every solution $(x, y, u)$ of $\Sigma$, and every solution $(\hat{x}, \hat{y}, u)$ of $\hat{\Sigma}$ the following $L_{2}$ output error bound holds true:

$$
\|y-\hat{y}\|_{2}^{2}<V(x(0), \hat{x}(0))+\sigma_{n}^{2}\|u\|_{2}^{2}
$$

where $V: X \times \mathbb{R}^{n-1} \rightarrow T$ is defined by

$$
V(x, \hat{x})=x_{o}^{T} \Lambda x_{o}+\sigma_{n}^{2} x_{c}^{T} \Lambda^{-1} x_{c}
$$

with $x_{o}=x-(\hat{x}, 0)=\left(x_{1}-\hat{x}, x_{2}\right)$ and $x_{c}=x+(\hat{x}, 0)=$ $\left(x_{1}+\hat{x}, x_{2}\right)$.

Before presenting the proof of Theorem 3 we present the proof of Theorem 2 .

Proof: [Proof of Theorem 2] Let $\hat{\Sigma}_{0}$ be the balanced LSS $\Sigma_{b a l}$ from Step 5 of Procedure1 and let $\Lambda_{0}=\Lambda$. For all $i=0, \ldots, n-r$ if $\Sigma_{i}=\left(n-i,\left\{\left({ }^{i} A_{q},{ }^{i} \bar{B},{ }^{i} C\right) \mid q \in Q\right\},{ }^{i} \hat{\phi}\right)$, then define

$$
\hat{\Sigma}_{i+1}=\left(n-i-1,\left\{\left({ }^{i+1} \bar{A}_{q},{ }^{i+1} \bar{B},{ }^{i+1} C\right) \mid q \in Q\right\},{ }^{i+1} \hat{\phi}\right)
$$

and $\Lambda_{i+1}$ as follows:

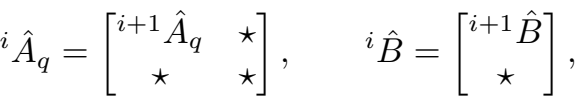

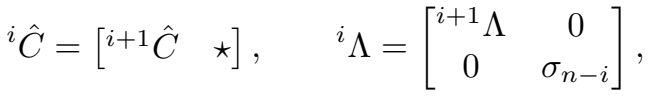

$$
\begin{aligned}
& { }^{i+1} \hat{\phi}(\zeta)={ }^{i} \hat{\phi}(\zeta, 0) \text {. }
\end{aligned}
$$

That is, $\hat{\Sigma}_{i+1}$ is obtained from $\hat{\Sigma}_{i}$ by applying Procedure 1 to $\hat{\Sigma}_{i}$ with grammians $\mathcal{Q}=\mathcal{P}=\Lambda_{i}$ and balancing transformation $\mathcal{S}$ being the identity. Note that $\hat{\Sigma}_{n-r}=\hat{\Sigma}$. It then follows that $\hat{\Sigma}_{0}$ is balanced, and as it is isomorphic with $\Sigma$, it is well-posed. Then by repeated application of Theorem 3 , it follows that $\hat{\Sigma}_{i}, i=1,2, \ldots, n-r$ are well posed, and balanced. For $u \in U$, let $\left({ }^{i} \hat{x}^{i}{ }^{i} \hat{y}, u\right)$ be solutions of $\hat{\Sigma}_{i}, i=0, \ldots, n-r$. From Theorem 3 it follows that

$$
\left\|{ }^{i} \hat{y}-{ }^{i+1} \hat{y}\right\|_{L_{2}}^{2} \leq V_{i}\left({ }^{i} \hat{x}(0),{ }^{i+1} \hat{x}(0)\right)+4 \sigma_{n-i}^{2}\|u\|_{L_{2}}^{2}
$$

where $V_{i}\left(\zeta_{1}, \zeta_{2}\right)=\zeta_{o}^{T} \Lambda_{i} \zeta_{o}+\sigma_{n-i}^{2} \zeta_{c} \Lambda_{i}^{-1} \zeta_{c}, \zeta_{c}=\zeta_{1}+$ $\left[\begin{array}{ll}\zeta_{2}^{T} & 0\end{array}\right]^{T}, \zeta_{o}=\zeta_{1}-\left[\begin{array}{ll}\zeta_{2}^{T} & 0\end{array}\right]^{T}$.

Let $(x, y, u)$ be a solution of $\Sigma$, with $x(0)=0$ and let $(\hat{x}, \hat{y}, u)$ be a solution of $\hat{\Sigma}$ with $\hat{x}(0)$. Let $\left({ }^{i} \hat{x},{ }^{i} \hat{y}, u\right)$ be solutions of $\hat{\Sigma}_{i}, i=1, \ldots, n-r-1$ such that ${ }^{i} \hat{x}(0)=0$ for all $i=1, \ldots, n-r-1$. Define $\left({ }^{0} \hat{x},{ }^{0} \hat{y}, u\right)=(\mathcal{S} x, y, u)$ and $\left({ }^{n-r} \hat{x},{ }^{n-r} \hat{y}, u\right)=(\hat{x}, \hat{y}, r)$. Then $\left({ }^{0} \hat{x},{ }^{0} \hat{y}, u\right)$ is a solution of $\hat{\Sigma}_{0}=\Sigma_{b a l}$ and $\left({ }^{n-r} \hat{x},{ }^{n-r} \hat{y}, u\right)$ is a solution of $\hat{\Sigma}_{n-r}=\hat{\Sigma}$, and hence for 10 holds. Moreover, since ${ }^{i} \hat{x}(0)=0$ for all $i=0, \ldots, n-r$, it follows that $V_{i}\left({ }^{i} \hat{x}(0),{ }^{i+1} \hat{x}(0)\right)=0$ for $i=0, \ldots, n-r-1$. It then follows that

$$
\begin{aligned}
& \|y-\hat{y}\|_{L_{2}}=\left\|\sum_{i=0}^{n-r-1}\left({ }^{i} \hat{y}-{ }^{i+1} \hat{y}\right)\right\|_{L_{2}} \\
& \leq \sum_{i=0}^{n-r-1}\left\|\left({ }^{i} \hat{y}-{ }^{i+1} \hat{y}\right)\right\|_{L_{2}} \leq\left(2 \sum_{i=r+1}^{n} \sigma_{i}\right)\|u\|_{L_{2}} .
\end{aligned}
$$

The proof of Theorem 3 follows from a sequence of lemmas, which we formulate and prove next.

Lemma 1: The LSS $\hat{\Sigma}$ is well-posed if $\Sigma$ is well-posed.

Proof: [Proof of Lemma 1] Consider the map

$$
\hat{G}(\zeta, t)=\left\{\nu \in \mathbb{R}^{n-1} \mid \nu=\hat{A}_{q} \zeta+\hat{B} u(t), q \in \hat{\phi}(z)\right\} .
$$


Upper semi-continuity of $\hat{G}$ follows from (the proof of) proposition 1.4 .14 in [37, p. 47] since $G$ from (4) is upper semi-continuous with finite (hence compact) values,

$$
f: \operatorname{Grap}(G) \rightarrow \mathbb{R}^{n-1} ;(x, t, v) \mapsto \nu=\left(v_{1}, \ldots, v_{n-1}\right)^{T}
$$

is continuous and $\hat{G}(\zeta, t)=\{f(\zeta, 0, t, v)\}_{v \in G(\zeta, 0, t)}$. Boundedness of $\hat{G}$ follows from the fact that by boundedness of $G$, there exists $M$ such that for all $(x, t, v) \in \operatorname{Grap}(G)$, $\|v\|_{2}<M$, hence $\|f(x, t, v)\|_{2}=\left\|\left(v_{1}, \ldots, v_{n-1}\right)^{T}\right\|_{2} \leq$ $\|v\|_{2}<M$.

Lemma 2: The LSS $\hat{\Sigma}$ is balanced. Moreover, $\Lambda_{1}$ is an observability and controllability grammian of $\hat{\Sigma}$.

Proof: [Proof of Lemma 2] We will start by showing that for any $\hat{\zeta}_{i} \in \mathbb{R}^{n-1}, q_{i} \in \hat{\phi}\left(\hat{\zeta}_{i}\right), i=1,2 u \in U$,

$$
\left(\hat{A}_{q_{1}} \hat{\zeta}_{1}+\hat{A}_{q_{2}} \hat{\zeta}_{2}+2 \hat{B} u\right)^{T} \Lambda_{1}^{-1}\left(\hat{\zeta}_{1}+\hat{\zeta}_{2}\right)-2\|u\|^{2}<0
$$

Notice that $q_{i} \in \hat{\phi}\left(\hat{\zeta}_{i}\right)$ if and only if $q_{i} \in \phi\left(\hat{\zeta}_{i}, 0\right)$. Since $\Lambda$ is a controllability grammian, the inequality (6) holds for $\zeta_{i}=\left[\begin{array}{ll}\hat{\zeta}_{i}^{T} & 0\end{array}\right]^{T}$ and $q_{i} \in \phi\left(\zeta_{i}\right), i=1,2$. Notice that $\Lambda^{-1}=$ $\operatorname{diag}\left(\Lambda_{1}^{-1}, \sigma_{n}^{-1}\right)$,

$$
\begin{aligned}
& A_{q_{1}} \zeta_{1}+A_{q_{2}} \zeta_{2}+2 B u=\left[\begin{array}{c}
\hat{A}_{q_{1}} \hat{\zeta}_{1}+\hat{A}_{q_{2}} \hat{\zeta}_{2}+2 \hat{B} u \\
\star
\end{array}\right] \\
& \text { and } \zeta_{1}+\zeta_{2}=\left[\begin{array}{cc}
\hat{\zeta}_{1}^{T}+\hat{\zeta}_{2}^{T} & 0
\end{array}\right]^{T} \text {. Hence } \\
& \left(A_{q_{1}} \zeta_{1}+A_{q_{2}} \zeta_{2}+2 B u\right)^{T} \Lambda^{-1}\left(\zeta_{1}+\zeta_{2}\right) \\
& =\left(\hat{A}_{q_{1}} \hat{\zeta}_{1}+\hat{A}_{q_{2}} \hat{\zeta}_{2}+2 \hat{B} u\right)^{T} \Lambda_{1}^{-1}\left(\hat{\zeta}_{1}+\hat{\zeta}_{2}\right) \text {. }
\end{aligned}
$$

This equality and (6) imply (11). For observability, we proceed exactly as above and use

$$
\left(\zeta_{1}-\zeta_{2}\right)^{T} C^{T} C\left(\zeta_{1}-\zeta_{2}\right)=\left(\hat{\zeta}_{1}-\hat{\zeta}_{2}\right)^{T} \hat{C}^{T} \hat{C}\left(\hat{\zeta}_{1}-\hat{\zeta}_{2}\right)
$$

to conclude

$$
\begin{aligned}
\left(\hat{A}_{q_{1}} \hat{\zeta}_{1}-\hat{A}_{q_{2}} \hat{\zeta}_{2}\right)^{T} \Lambda_{1}^{-1}\left(\hat{\zeta}_{1}+\hat{\zeta}_{2}\right) & \\
& +\left(\hat{\zeta}_{1}-\hat{\zeta}_{2}\right)^{T} \hat{C}^{T} \hat{C}\left(\hat{\zeta}_{1}-\hat{\zeta}_{2}\right)<0
\end{aligned}
$$

for any $\hat{\zeta}_{i} \in \mathbb{R}^{n-1}$ and $q_{i} \in \hat{\phi}\left(\hat{\zeta}_{i}\right), i=1,2$.

Lemma 3: For every $u \in U, \zeta_{1}, \zeta_{2} \in \mathbb{R}^{n}$ and for every $\mathcal{A}_{i}$ such that $\mathcal{A}_{i} \in \operatorname{co}\left\{A_{q} \mid q \in \phi\left(\zeta_{i}\right)\right\}, i=1,2$

$$
\begin{aligned}
\left(\mathcal{A}_{1} \zeta_{1}-\mathcal{A}_{2} \zeta_{2}\right)^{T} \Lambda\left(\zeta_{1}-\zeta_{2}\right) & \\
& +\left(\zeta_{1}-\zeta_{2}\right)^{T} C^{T} C\left(\zeta_{1}-\zeta_{2}\right)<0
\end{aligned}
$$

$$
\left(\mathcal{A}_{1} \zeta_{1}+\mathcal{A}_{2} \zeta_{2}+2 B u\right)^{T} \Lambda^{-1}\left(\zeta_{1}+\zeta_{2}\right)-4\|u\|^{2}<0 .
$$

Proof: [Proof Lemma 3] Here, we will prove (12a); the proof of $12 \mathrm{~b}$ is identical. We consider the observability grammian in (6). We will show that for all $\zeta_{1}, \zeta_{2} \in X$, and for all $\alpha_{q_{1}}, \beta_{q_{2}},\left(q_{1}, q_{2}\right) \in \phi\left(\zeta_{1}\right) \times \phi\left(\zeta_{2}\right)$, such that $\sum_{q_{1} \in \phi\left(\zeta_{1}\right)} \alpha_{q_{1}}=1$ with $0 \leq \alpha_{q_{1}} \leq 1$ and similarly $\sum_{q_{2} \in \phi\left(\zeta_{2}\right)} \beta_{q_{2}}=1$ with $0 \leq \beta_{q_{2}} \leq 1$, the following inequality holds true

$$
\begin{aligned}
\left(\sum_{q_{1} \in \phi\left(\zeta_{1}\right)} \alpha_{q_{1}} A_{q_{1}} \zeta_{1}\right. & \left.-\sum_{q_{2} \in \phi\left(\zeta_{2}\right)} \beta_{q_{2}} A_{q_{2}} \zeta_{2}\right)^{T} \Lambda\left(\zeta_{1}-\zeta_{2}\right) \\
& +\left(\zeta_{1}-\zeta_{2}\right)^{T} C^{T} C\left(\zeta_{1}-\zeta_{2}\right)<0
\end{aligned}
$$

At the outset, we simplify the notation, and write $a_{q_{1}}=$ $A_{q_{1}} \zeta_{1} \Lambda\left(\zeta_{1}-\zeta_{2}\right), b_{q_{2}}=-A_{q_{2}} \zeta_{1} \Lambda\left(\zeta_{1}-\zeta_{2}\right)$, and $c=\left(\zeta_{1}-\right.$ $\left.\zeta_{2}\right)^{T} C^{T} C\left(\zeta_{1}-\zeta_{2}\right)$. Using the new notation, (5) becomes $a_{q_{1}}+b_{q_{2}}+c<0$. Subsequently, we have

$$
\begin{aligned}
0 & >\sum_{q_{2} \in \phi\left(\zeta_{2}\right)} \beta_{q_{2}}\left(\sum_{q_{1} \in \phi\left(\zeta_{1}\right)} \alpha_{q_{1}}\left(a_{q_{1}}+b_{q_{2}}+c\right)\right) \\
& =\sum_{q_{1}} \alpha_{q_{1}} a_{q_{1}}+\sum_{q_{2}} \beta_{q_{2}} b_{q_{2}}+c,
\end{aligned}
$$

which shows $(13)$.

Proof: [Proof Theorem 3] By Lemma 1] the LSS $\hat{\Sigma}$ is well-posed, and by Lemma 2, it is balanced. The result (9) will be obtained by a Lyapunov like argument. In other words, we will analyze the behavior of $V$ along the solutions via $\dot{V}=\frac{d}{d t} V(x(t), \hat{x}(t))$. To this end, consider first

$$
\begin{aligned}
\frac{d}{d t} x_{o}(t) & =\frac{d}{d t}\left(x(t)-\left[\begin{array}{c}
\hat{x}(t) \\
0
\end{array}\right]\right) \\
& =\sum_{q \in \phi(x(t))} \alpha_{q}(t)\left(A_{q} x(t)+B u(t)\right) \\
& -\sum_{\hat{q} \in \hat{\phi}(\hat{x}(t))}\left[\begin{array}{c}
\left.\hat{\alpha}_{\hat{q}}(t)\left(\hat{A}_{\hat{q}} \hat{x}(t)+\hat{B} u(t)\right)\right] \\
0
\end{array}\right] \\
= & \sum_{q \in \phi(x(t))} \alpha_{q}(t)\left(A_{q} x(t)+B u(t)\right) \\
- & \sum_{\hat{q} \in \hat{\phi}(\hat{x}(t))} \hat{\alpha}_{\hat{q}}(t)\left(A_{\hat{q}}\left[\begin{array}{c}
\hat{x}(t) \\
0
\end{array}\right]+B u\right) \\
& +\sum_{\hat{q} \in \hat{\phi}(\hat{x}(t))}\left[\begin{array}{c}
0 \\
\hat{\alpha}_{\hat{q}}(t)\left(A_{\hat{q}, 21} \hat{x}(t)+B_{2} u(t)\right)
\end{array}\right] .
\end{aligned}
$$

where $\alpha_{q}(t), \hat{\alpha}_{\hat{q}}(t) \in[0,1], \quad \sum_{q \in \phi(x(t))} \alpha_{q}(t)=1$, $\sum_{\hat{q} \in \hat{\phi}(\hat{x}(t))} \hat{\alpha}_{q}(t)=1$. In order to simplify the notation, we set $\alpha_{q}(t)=0$ and $\hat{\alpha}_{\hat{q}}(t)=0$ for $q \notin \phi(x(t))$ and $\hat{q} \notin \hat{\phi}(\hat{x}(t))$, and we drop the dependence on $t$ of $x_{o}(t), x(t), \hat{x}(t), u(t), \alpha_{q}(t), \hat{\alpha}_{\hat{q}}(t)$. With this in mind, using (12a), we get for the first term of $\dot{V}$

$$
\begin{aligned}
\frac{1}{2} \frac{d}{d t}( & \left.x_{o}^{T}(t) \Lambda x_{o}(t)\right) \\
= & \left(\sum_{q} \alpha_{q}\left(A_{q} x+B u\right)-\sum_{\hat{q}} \hat{\alpha}_{\hat{q}}\left(A_{\hat{q}}\left[\begin{array}{l}
\hat{x} \\
0
\end{array}\right]+B u\right)\right)^{T} \Lambda x_{o} \\
& \quad+\sum_{\hat{q}} \hat{\alpha}_{\hat{q}}\left(A_{\hat{q}, 21} \hat{x}+B_{2} u\right)^{T} \sigma_{n} x_{2} \\
<- & x_{o}^{T} C^{T} C x_{o}+\sum_{\hat{q}} \hat{\alpha}_{\hat{q}}\left(A_{\hat{q}, 21} \hat{x}+B_{2} u\right)^{T} \sigma_{n} x_{2} \\
= & -\|y-\hat{y}\|^{2}+\sum_{\hat{q}} \hat{\alpha}_{\hat{q}}\left(A_{\hat{q}, 21} \hat{x}+B_{2} u\right)^{T} \sigma_{n} x_{2} .
\end{aligned}
$$


To estimate the second term of $\dot{V}$ we proceed, as above by first calculating

$$
\begin{aligned}
\frac{d}{d t} x_{c}= & \frac{d}{d t}\left(x+\left[\begin{array}{l}
\hat{x} \\
0
\end{array}\right]\right) \\
= & \sum_{q \in \phi(x)} \alpha_{q}\left(A_{q} x+B u\right)+\sum_{\hat{q} \in \hat{\phi}(\hat{x})}\left[\begin{array}{c}
\hat{\alpha}_{\hat{q}}\left(\hat{A}_{\hat{q}} \hat{x}+\hat{B} u\right) \\
0
\end{array}\right] \\
= & \sum_{q} \alpha_{q}\left(A_{q} x+B u\right)+\sum_{\hat{q}} \hat{\alpha}_{\hat{q}}\left(A_{\hat{q}}\left[\begin{array}{l}
\hat{x} \\
0
\end{array}\right]+B u\right) \\
& -\sum_{\hat{q}}\left[\begin{array}{c}
0 \\
\hat{\alpha}_{\hat{q}}\left(A_{\hat{q}, 21} \hat{x}+B_{2} u\right)
\end{array}\right] .
\end{aligned}
$$

Note that in the expression above we dropped the dependent on $t$ of $x_{c}(t), x(t), \hat{x}(t), u(t), \alpha_{q}(t), \hat{\alpha}_{\hat{q}}(t)$, and $\alpha_{q}(t), \hat{\alpha}_{\hat{q}}(t)$ are the same as in the expression for $\frac{d}{d t} x_{o}$. Hence, using (12b), we get for the second term of $\dot{V}$

$$
\begin{aligned}
& \frac{1}{2} \frac{d}{d t}\left(\sigma_{n}^{2} x_{c}^{T} \Lambda^{-1} x_{c}\right) \\
& =\sigma_{n}^{2}\left(\sum_{q} \alpha_{q}\left(A_{q} x+B u\right)\right. \\
& \left.\quad+\sum_{\hat{q}} \hat{\alpha}_{\hat{q}}\left(A_{\hat{q}}\left[\begin{array}{c}
\hat{x} \\
0
\end{array}\right]+B u\right)\right)^{T} \Lambda^{-1} x_{c} \\
& \quad-\sum_{\hat{q}} \hat{\alpha}_{\hat{q}}\left(A_{\hat{q}, 21} \hat{x}+B_{2} u\right)^{T} \sigma_{n} x_{2} \\
& <\sigma_{n}^{2}\|u\|^{2}-\sum_{\hat{q}} \hat{\alpha}_{\hat{q}}\left(A_{\hat{q}, 21} \hat{x}+B_{2} u\right)^{T} \sigma_{n} x_{2} .
\end{aligned}
$$

In summary, we have the estimate

$$
\dot{V}<-\|y-\hat{y}\|^{2}+\sigma_{n}^{2}\|u\|^{2} .
$$

Integrating 14 from 0 to $t$, using that $V>0$, and taking the limit $t \rightarrow \infty$ yields 9 .

\section{EXAMPLE}

In this illustrative example, we consider a simplified version of the partitioning in Fig 1 . where only $p_{1}=1$ is considered (we define $q=1$ when $p_{1} \leq 1$ and $q=2$ when $K \geq p_{1} \geq 1$ and $q=3$ when $p_{1} \geq K, K=10^{5}$ ). The linear systems are given by

$$
\begin{aligned}
A_{1} & =\left[\begin{array}{cc}
-1 & 1 \\
0 & -1
\end{array}\right], \quad A_{2}=\left[\begin{array}{cc}
-2 & 1 \\
0 & -4
\end{array}\right], A_{3}=0 \\
B & =\left[\begin{array}{ll}
1 & 3
\end{array}\right]^{T}, \quad C=\left[\begin{array}{ll}
1 & 1
\end{array}\right] .
\end{aligned}
$$

Applying the steps in Procedure 1, results in the following common grammian

$$
\Lambda=\left[\begin{array}{cc}
16.1092 & 0 \\
0 & 11.7295
\end{array}\right] .
$$

Subsequently, the reduced order model is given by

$$
\begin{aligned}
\hat{A}_{1} & =-0.4854, \hat{A}_{2}=-2.4864, \hat{A}_{3}=0 \\
\hat{B} & =-3.8028, \hat{C}=-1.0448 .
\end{aligned}
$$

Fig. 2 illustrates the input signal (top plot) and the resulting output from the original and reduced system (bottom plot).
Further, Fig. 3 shows the original switched system's full state trajectory (left), along with the last $10 \mathrm{sec}$. of the trajectory (right), illustrating that the system converges to the equilibrium point, when the input is zero. Furthermore, the values for the error bound, from (8), becomes

$$
\begin{aligned}
\|y-\hat{y}\|_{L_{2}} & =6.7962, \\
2 \sigma_{1}\|u\|_{L_{2}} & =909.4657 .
\end{aligned}
$$
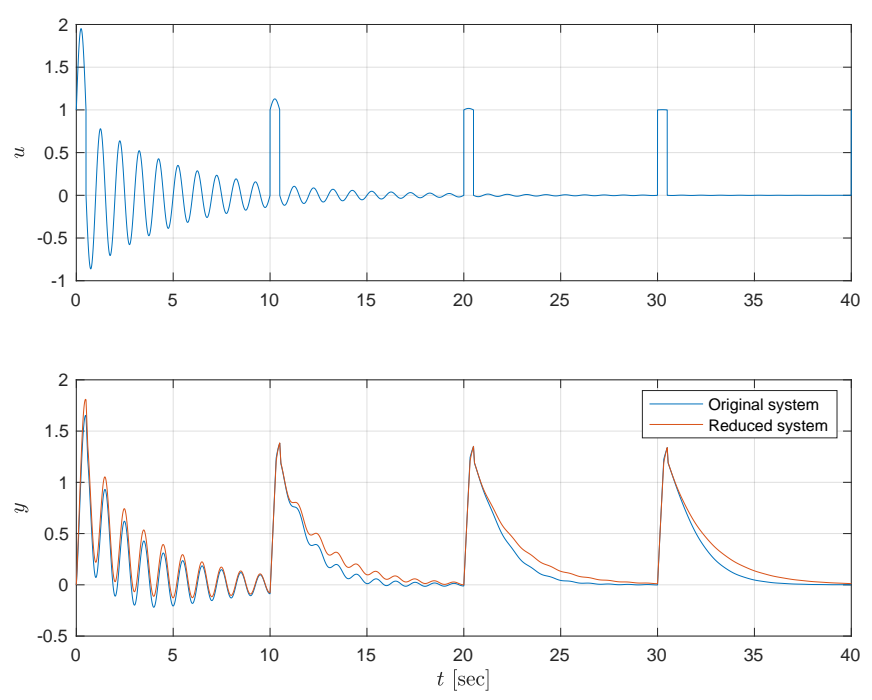

Fig. 2. Input signal (top) and output of the original and reduced system (bottom). The reduced system has a 78\% goodness of fit according to the Matlab function goodnessOfFit(), evaluated with a NRMSE cost function.
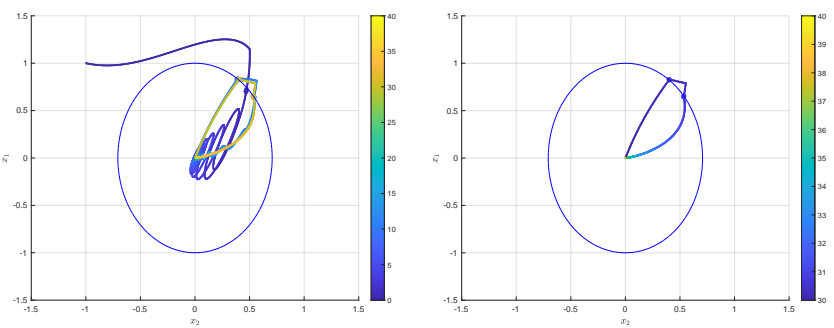

Fig. 3. Full state trajectory of the original switched system (left). Last 10 seconds of the state trajectory (right). Colorbar represents time ( $t$ [sec]) and ' $*$ ' indicates switching. From the figure on the right, it is seen that the system indeed converges to the equilibrium point, when the input is zero.

It is remarked that the input signal is a linear combination of a damped sinusoidal and 6 unit-steps ( 3 up and 3 down). We therefore have a discontinuous input signal which strictly speaking is not admissible. However, from (18) and (19) we see that the conclusion from Theorem 2 still holds true. One could of course just approximate each pair of unit-steps (1 up and 1 down) with a bump function to obtain an admissible input signal, yielding almost identical results as one can approximate to arbitrary precision.

\section{CONCLUSIONS}

We have presented a model reduction algorithm based on balanced truncation for a large class of piecewise-affine 
hybrid systems. We have also formulated an analytic error bound for the difference between the output of the reducedorder model and the original one. This error bound is a direct counterpart of the well-known error bound for balanced truncation of linear systems. Future research will be directed towards extending these results to systems where the $B$ and $C$ matrices depend on the discrete mode and where statejumps are present. Another possible research direction is to derive error bounds in general $L_{p}$ norms for $p \neq 2$. Finally, we plan to analyze the computational complexity of the proposed method and test it on larger examples.

\section{REFERENCES}

[1] D. Liberzon, Switching in Systems and Control. Birkhäuser, Boston, 2003.

[2] Z. Sun and S. Ge, Stability Theory of Switched Dynamical Systems. Springer, 2011.

[3] R. Goebel, R. G. Sanfelice, and A. R. Teel, Hybrid Dynamical Systems: Modeling, Stability, and Robustness. Princeton University Press, 2012.

[4] A. C. Anthoulas, Approximation of Large-Scale Dynamical Systems. SIAM, 2005.

[5] H. Gao, J. Lam, and C. Wang, "Model simplification for switched hybrid systems," Systems and Control Letters, vol. 55, pp. 1015-1021, 2006.

[6] L. Zhang, E. Boukas, and P. Shi, “ $\mu$-Dependent model reduction for uncertain discrete-time switched linear systems with average dwell time," International Journal of Control, vol. 82, no. 2, pp. 378-388, 2009.

[7] L. Zhang, P. Shi, E. Boukas, and C. Wang, "H-infinity model reduction for uncertain switched linear discrete-time systems," Automatica, vol. 44, no. 8, pp. 2944-2949, 2008.

[8] L. Zheng-Fan, C. Chen-Xiao, and D. Wen-Yong, "Stability analysis and $H_{\infty}$ model reduction for switched discrete-time time-delay systems," Mathematical Problems in Engineering, vol. 15, 2014.

[9] N. Monshizadeh, H. L. Trentelman, and M. K. Camlibel, "A simultaneous balanced truncation approach to model reduction of switched linear systems," IEEE Transactions on Automatic Control, vol. 57, no. 12, pp. 3118-3131, 2012.

[10] A. Birouche, J. Guilet, B. Mourillon, and M. Basset, "Gramian based approach to model order-reduction for discrete-time switched linear systems," in Proceedings of the 18th Mediterranean Conference on Control and Automation, 2010, pp. 1224-1229.

[11] A. Birouche, B. Mourllion, and M. Basset, "Model reduction for discrete-time switched linear time-delay systems via the $H_{\infty}$ stability," Control and Intelligent Systems, vol. 39, no. 1, pp. 1-9, 2011.

[12] —, "Model order-reduction for discrete-time switched linear systems," Int. J. Systems Science, vol. 43, no. 9, pp. 1753-1763, 2012.

[13] I. V. Gosea, M. Petreczky, and A. C. Antoulas, "Balanced truncation for linear switched systems," Advances in Computational Mathematics, 2018.

[14] H. R. Shaker and R. Wisniewski, "Generalized gramian framework for model/controller order reduction of switched systems," International Journal of Systems Science, vol. 42, no. 8, pp. 1277-1291, 2011.

[15] H. Shaker and R. Wisniewski, "Model reduction of switched systems based on switching generalized gramians," International Journal of Innovative Computing, Information and Control, vol. 8, no. 7(B), pp. 5025-5044, 2012.

[16] M. Petreczky, R. Wisniewski, and J. Leth, "Balanced truncation for linear switched systems," Nonlinear Analysis: Hybrid Systems, vol. 10, pp. 4-20, Nov. 2013.

[17] P. Schulze and B. Unger, "Model reduction for linear systems with low-rank switching," SIAM J. Control Optim., vol. 56, no. 6, pp. 43654384, 2018.

[18] I. Pontes Duff, S. Grundel, and P. Benner. (2018, June) New Gramians for switched linear systems: reachability, observability, and model reduction. available online at https://arxiv.org/abs/1806.00406 accepted for publication in IEEE Trans. Auto. Control.

[19] G. Kotsalis, "Model reduction for hidden markov models," Ph.D. dissertation, Massachusets Institute of Technology, 2006.
[20] G. Kotsalis, A. Megretski, and M. A. Dahleh, "Balanced truncation for a class of stochastic jump linear systems and model reduction of hidden Markov models," IEEE Transactions on Automatic Control, vol. 53, no. 11, 2008.

[21] M. Bastug, M. Petreczky, R. Wisniewski, and J. Leth, "Reachability and observability reduction for linear switched systems with constrained switching," Automatica, vol. 74, pp. 162-170, 2016.

[22] — - "Model reduction by moment matching for linear switched systems," IEEE Transactions on Automatic Control, vol. 61, pp. 34223437, 2016.

[23] M. Bastug, "Model reduction of linear switched systems and lpv statespace models," Ph.D. dissertation, Aalborg University, 2016.

[24] I. V. Gosea, M. Petreczky, and A. C. Antoulas, "Data-driven model order reduction of linear switched systems in the loewner framework," SIAM Journal on Scientific Computing, Society for Industrial and Applied Mathematics, vol. 40, no. 2.

[25] A. V. Papadopoulos and M. Prandini, "Model reduction of switched affine systems," Automatica, vol. 70, pp. 57-65, 2016.

[26] G. Scarciotti and A. Astolfi, "Model reduction for hybrid systems with state-dependent jumps," IFAC-PapersOnLine, vol. 49, no. 18, pp. 850 $-855,2016$.

[27] G. Scarciotti, A. R. Teel, and A. Astolfi, "Model reduction for linear differential inclusions: moment-set and time-variance," pp. 3483-3487, May 2017.

[28] B. Besselink, "Model reduction for nonlinear control systems : with stability preservation and error bounds," Ph.D. dissertation, Department of Mechanical Engineering, 2012.

[29] M. Petreczky and J. H. van Schuppen, "Observability reduction of piecewise-affined hybrid systems," in Proceedings 19th International Symposium on Mathematical Theory of Networks and Systems, 2010, pp. 203-210.

[30] A. C. Antoulas and D. C. Sorensen, "Approximation of large-scale dynamical systems: an overview," Int. J. Appl. Math. Comput. Sci., vol. 11, no. 5, pp. 1093-1121, 2001, numerical analysis and systems theory (Perpignan, 2000).

[31] M. Petreczky, R. Wisniewski, and J. Leth, "Balanced truncation for linear switched systems," Nonlinear Analysis: Hybrid Systems, vol. 10, pp. $4-20,2013$, special Issue related to IFAC Conference on Analysis and Design of Hybrid Systems (ADHS 12). [Online]. Available: http://www.sciencedirect.com/science/article/pii/S1751570X13000150

[32] M. Petreczky, "Realization theory of linear and bilinear switched systems: A formal power series approach, part i," ESAIM COCV, vol. 17, pp. 410-445, 2011.

[33] — , "Realization theory of hybrid systems," Ph.D. dissertation, Vrije Universiteit, Amsterdam, 2006.

[34] J. Leth and R. Wisniewski, "On formalism and stability of switched systems," Journal of Control Theory and Applications, vol. 10, no. 2, pp. 176-183, May 2012. [Online]. Available: https://doi.org/10.1007/s11768-012-0138-3

[35] A. F. Filippov, Differential equations with discontinuous righthand sides, ser. Mathematics and its Applications (Soviet Series). Kluwer Academic Publishers Group, Dordrecht, 1988, vol. 18, translated from the Russian. [Online]. Available: https://doi.org/10. 1007/978-94-015-7793-9

[36] J.-P. Aubin and A. Cellina, Differential inclusions, ser. Grundlehren der Mathematischen Wissenschaften [Fundamental Principles of Mathematical Sciences]. Springer-Verlag, Berlin, 1984, vol. 264, set-valued maps and viability theory. [Online]. Available: https: //doi.org/10.1007/978-3-642-69512-4

[37] J.-P. Aubin and H. Frankowska, Set-valued analysis, ser. Modern Birkhäuser Classics. Birkhäuser Boston, Inc., Boston, MA, 2009, reprint of the 1990 edition [MR1048347]. [Online]. Available: https://doi.org/10.1007/978-0-8176-4848-0 